\title{
Successful Pregnancy Complicated by Adnexal Torsion after IVF in a 45-Year- Old Woman
}

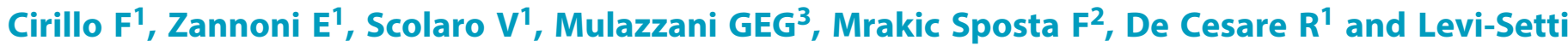 \\ $\mathrm{PE}^{1 *}$
}

1Department of Gynaecology, Division of Gynaecology and Reproductive Medicine, Humanitas Fertility Center, EBCOG/ESHRE Subspecialty European Center in Reproductive Medicine, Humanitas Research Hospital, Rozzano (Milan), Italy

2Humanitas University, Humanitas Research Hospital, Rozzano, Milan, Italy

3Department of Radiology, Division of Diagnostic Radiology, Humanitas Research Hospital, Rozzano (Milan), Italy

*Corresponding author: Paolo Emanuele Levi-Setti, Department of Gynaecology, Division of Gynaecology and Reproductive Medicine, Humanitas Fertility Center, EBCOG/ESHRE Subspecialty European Center in Reproductive Medicine, Humanitas Research Hospital, Rozzano (Milan), Italy, Tel: 10125410158, E-mail: paolo.levi_setti@humanitas.it

Received date: 12 June, 2016; Accepted date: 26 August, 2016; Published date: 29 August, 2016

Citation: Cirillo F, Zannoni E, Scolaro V. Successful pregnancy complicated by adnexal torsion after IVF in a 45-year-old woman, Gynecol Obstet Case Rep. 2016, 2:2.

\section{Abstract}

Ovarian torsion accounts for $3 \%$ of gynecological emergencies. Its incidence is higher in all those cases of ovarian hypermobility and adnexal masses, such as Ovarian Hyperstimulation Syndrome (OHSS) as a consequence of in vitro fertilization (IVF) treatments. It is an acute condition; its diagnostic work-up is difficult because of its vague clinical presentation. Nonetheless its diagnosis must be as rapid as possible because a prompt surgical detorsion is the only treatment assuring ovarian function recovery. The diagnostic iter includes complete cell blood count, D-dimer, transabdominal ultrasound (US), transvaginal US and magnetic resonance (MRI).

Our patient was a 45-year-old woman with good ovarian reserve indexes. She underwent a second level IVF stimulation because of a male infertility factor. Despite her age she developed an OHSS. Furthermore, twenty minutes after the embryo transfer, a right ovarian torsion occurred; the definitive diagnosis was made thanks to TV US and abdominal MRI. The patient was immediately treated with laparoscopic detorsion and after two weeks from the embryo transfer her beta HCG were positive with regular duplication times.

The significance of this case stands in the chosen diagnostic iter, which actually underlines the importance of MRI in the prevention of useless surgeries, and in the patient's clinical peculiarity: despite the advanced age she developed an OHSS and, despite the ovarian torsion and the surgery, she successfully got pregnant.

Keywords: Ovarian torsion; IVF; Diagnosis and treatment

\section{Introduction}

Ovarian torsion occurs when the ovarian vascular pedicle performs a complete or partial rotation around its axis with consequent impairment in vascular supply [1].

Torsion is considered the 5th most common surgical emergency in women, accounting for more than $3 \%$ of all gynaecological emergencies [2]. It has a trimodal age distribution with $80 \%$ of all the cases occurring in early childhood, reproductive age (15-30 years), especially in early pregnancy, and the remaining in the post-menopausal period [2]. This peculiar epidemiological setting is strictly related to the pathogenesis of the condition itself; the main causes of torsion are the hypermobility of the ovary (less than $40 \%$ of cases) and the presence of adnexal masses (more than 60\%) [2]. Ovarian stimulation and ovarian hyperstimulation syndrome (OHSS) are also associated with an increased risk because of the cystic enlargement of the ovary [3]. Among the women undergoing in vitro fertilization (IVF), the incidence of adnexal torsion ranges from $0.08 \%$ to $0.13 \%$ [3]. In general, there is a slight right predominance $(55 \%)$ because of the differences between the right and left adnexa in terms of vascular anatomy and because of the presence of the sigmoid colon on the left; in $10 \%$ of cases there can be also a concomitant torsion of the contralateral ovary $[1,4]$.

As already said, torsion is a surgical emergency in the sense that, if not timely corrected, persistent vascular occlusion results in infarction and necrosis. Therefore, prompt diagnosis and surgical restoration of the blood supply are essential for salvage of the tube and ovary.

The main problem in the diagnostic work-up is that the clinical presentation of ovarian torsion is vague and characterized by nonspecific signs and symptoms. Torsion symptoms include lower abdominal pain, acute or chronic, intermittent or constant, nausea and emesis; signs include a 
palpable pelvic mass, localized peritoneal irritation, a lowgrade fever, and leukocytosis $[2,5]$.

A valid support in the diagnostic process is given by pelvic ultrasonography (US) with Doppler evaluation; although Doppler evaluation provides a good analysis of adnexal perfusion and vascularity, it has a high specificity but a low sensitivity. On the other side CT and MRI can give further information: CT and MRI can show uterine deviation, pelvic fat infiltration, high-density or signal-specific hematoma, thickened fallopian tubes, lack of enhancement sign $[4,5]$.

A third diagnostic test demonstrated to be valid in the evaluation of a suspected ovarian torsion is the D-dimer quantification. The clinical association between $\mathrm{D}$-dimer values and ovarian torsion has been studied in animal models since 2011 and later retrospective studies have then concluded that this parameter may be used for the early diagnosis of adnexal torsion $[6,7]$.

Once diagnosed, conservative ovarian detorsion is the preferred management option in children, adolescents and reproductive aged women. In general, detorsion has been demonstrated to save more than $90 \%$ of these ovaries with consequent ovarian function recovery [3]. On the other side, salpingo-ophrectomy is the approach of choice for women in post-menopausal age, with suspect of malignancy or with nonviable ovary [1].

\section{Case Description}

In November 2014 a healthy 45-year-old woman with silent past medical history was referred to our center because of a problem of male infertility. She had had a previous spontaneous pregnancy with a different partner in year 2000 ended in abortion.

Despite her age, the patient showed good ovarian reserve indexes: 22 antral follicles, FSH $5.3 \mathrm{IU} / \mathrm{mL}$ and $\mathrm{AMH} 2.5 \mathrm{ng} / \mathrm{ml}$.

An intra-cytoplasmic sperm injection (ICSI) procedure was performed because of the male indication. She received oral estrogen-progestin therapy for one month; after the proper suppression was achieved, controlled ovarian hyperstimulation was initiated with recombinant FSH ( $r F S H)$ (Gonal-F, Serono Inc.) at the daily dose of 225 IU. On day fifth of stimulation, recombinant LH (Luveris, Serono Inc.) was added 75 IU daily, while the antagonist (Orgalutran, MSD) was started on day six at a daily dose of $250 \mathrm{IU}$.

After 11 days of stimulation, she showed signs of impending risk of OHSS: 19 follicles of more than $10 \mathrm{~mm}$ in diameter and high levels of estradiol $(4822 \mathrm{pg} / \mathrm{mL}$ ). In order to control the OHSS risk, the ovulation was induced with $0.2 \mathrm{mg}$ of $\mathrm{GnRH}$ agonist (Decapeptyl, Ipsen SPA) in single administration 34 hours before oocyte retrieval.

At oocyte pick up 18 oocytes were retrieved; since the postoperative conditions were optimal, the patient was sent home after the routine time of observation. A rescue protocol was planned: chorionic gonadotropin (Gonasi, IBSA) 1500 IU and progesterone (Crinone, Watson) plus estradiol valerate (Progynova, Bayer) for luteal phase support [8].

During the second day after surgery, the patient entered the ER of another hospital because of a sudden pelvic pain; she had signs of mild OHSS. She was monitored for $12 \mathrm{~h}$.

On day three after surgery, the patient was routinely evaluated in our center in order to perform the embryo transfer: she was completely asymptomatic. She underwent a transvaginal US examination which showed $19 \mathrm{~mm}$ of free fluid in the pelvis and enlarged ovaries: $80 \mathrm{~mm} \times 62 \mathrm{~mm}$ on the right and $84 \mathrm{~mm} \times 62 \mathrm{~mm}$ on the left. Given the reassuring clinical situation of the woman, three embryos were decided to be transferred under trans-abdominal US guidance using a soft catheter (Wallace Sure View Embryo Replacement Catheter; Smith Medical).

Twenty minutes after the ET, the patient started complaining about a severe intermittent pelvic pain in the right iliac fossa; the pain showed not to be responsive to pain killers and it was accompanied by nausea and bile-stained vomit. Because of the worsening of symptoms, the progressive increase in leukocytosis without a concomitant appearance of anaemia, the patient underwent a transvaginal US. The ultrasonographic examination showed a mild pelvic free fluid accumulation; the right ovary was not visible at all making the picture very suspicious for ovarian dislocation. After that, since the US picture was non detrimental, an MRI was prescribed. The magnetic resonance images documented the presence of signs of recent ovarian stimulation, a slight ovarian asymmetry (right ovary $94 \mathrm{~mm} \times 63 \mathrm{~mm}$, left ovary $55 \mathrm{~mm} \times 87 \mathrm{~mm}$ ) and a very high T2 and T1 fat-saturated (FS) sequence signal in the right ovary. According to the clinical context, this latter feature together with a thickened and edematous right ovarian pedicle, recognizable before the psoas muscle, were highly indicative of blood stasis from a haemorrhagic spot within the ovary (Figure 1). The radiologist described the picture as highly suggestive of right ovarian torsion.

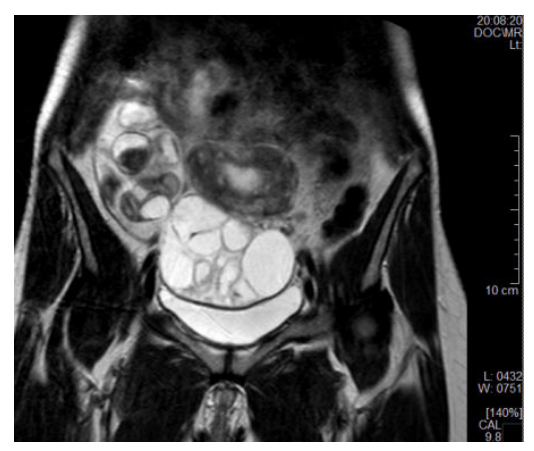

Figure 1 RMI image showing ovaries asymmetry and signs of torsion

D-dimer was not evaluated in our patient because this clinical parameter at that time was not yet in our standard diagnostic iter.

Finally, an emergency laparoscopy was performed for a diagnostic confirmation and an early intervention. Both the 
ovaries were three times their normal volume, with many corpora lutea, and the right adnexa was twisted about its axis, dislocated in the extra-pelvic area and with necrotic tissue cephalad (Figure 2). Careful inspection of the whole pelvis and right ovarian detorsion were carried out: the necrotic tissue ( $1 / 3$ of the entire right ovary) was debrided and extracted through endobag and sent for histological examination.

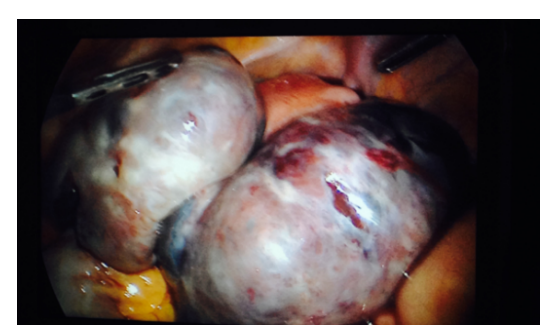

Figure $\mathbf{2}$ Laparoscopic view showed a right ovarian torsion

The post-operative course was eventless. After two weeks from the ET the patient showed positive beta HCG with correct doubling time. The patient continued a standard luteal phase support with $200 \mathrm{mg}$ vaginal progesterone for 12 weeks.

Pregnancy evolved uneventfully, with regular follow up.

At 38 weeks of gestation, she delivered with obstetric vacuum for cardiotocographic alteration a healthy female baby of $2900 \mathrm{~g}$.

Today, the patient still has one cryopreserved blastocyst.

\section{Discussion}

When our patient was finally diagnosed with ovarian torsion, her case immediately gathered great attention because of its unicity. Many aspects of the woman's clinical picture were peculiar both if considered alone and if taken all together in the general view.

First of all, the woman at the time of treatment initiation was 45 years old and, despite her age, she had very high ovarian reserve indexes. After the induction cycle, indeed, she was at high risk of hyperstimulation syndrome. She actually developed an OHSS and, interestingly, she experienced hyperstimulation even if she had been treated with a pharmacological protocol designed to reduce such a risk: antagonist stimulation cycle together with GnRh agonist Decapeptyl induction. This is interesting because it corresponds to the recent literature researches demonstrating that in facts this pharmacological treatment does not reduce the OHSS risk as much as it was thought to [9].

The choice of using Decapeptyl as induction trigger and to send the patient to embryo transfer regardless of the high stimulation response lies in the clinical protocol in use in our structure.

The known risk factors for the OHSS are a low BMI (<18), high ovarian reserve indexes, first IVF cycle or previous OHSS, high response to induction treatments in terms of number of follicles ( $>25$ ) and high estrogen levels (at a lesser extent). Our protocols, however, according to what demonstrated by the updated literature, take into consideration for the evaluation of the OHSS risk only the response to the induction treatment in terms of number of recruited follicles. Our criteria to choose which trigger to use and to decide whether to abort the cycle and postpone the embryo transfer or to perform it thus depend only on the response on the day of trigger administration and the number of oocytes retrieved:

- $\leq 18$ follicles with $>12 \mathrm{~mm}$ of diameter: trigger with $\mathrm{rHCG}$, successive embryo transfer and luteal phase support with intravaginal progesterone $200 \mathrm{mg}$ three times a day or Crinone progesterone $8 \%$ twice a day from the day of oocyte retrieval;

- 19-25 follicles with $>12 \mathrm{~mm}$ of diameter: trigger with $\mathrm{GnRH}$ agonist triptorelin, Decapeptyl $0.2 \mathrm{mg}$ vials s.c., plus HCG, Gonasi $1500 \mathrm{IU}$, administered in the operating room on the day of oocyte pickup, successive embryo transfer and luteal phase support with Crinone $8 \%$ once a day or Prometrium/ Progeffik $200 \mathrm{mg}$ twice a day plus Progynova $4 \mathrm{mg}$ (2 Tablets/die);

- >25 follicles with >12 mm of diameter and >20 retrieved oocytes: trigger with triptorelin, Decapeptyl $0.2 \mathrm{mg} 2$ vials s.c., and elective cryopreservation.

In the second place, the woman's clinical picture was peculiar because, although she experienced a severe ovarian torsion with a consequent invasive therapeutic pelvic intervention, she succeeded in getting pregnant on her first stimulation cycle and she successfully delivered a healthy baby girl [3].

Third, the patient's picture and the adopted clinical approach mirrored what is described in the present literature for ovarian torsion presentation and management.

In this sense, away from its peculiarities, this case report can be used to clearly exemplify the practical diagnostic method employed in patients with ovarian torsion.

From the point of view of the clinical presentation, her ovarian torsion corresponds to what is described in the present literature: lower abdominal pain, nausea, emesis, together with signs of localized peritoneal irritation, low-grade fever and leukocytosis. She also responds to the five criteria used for the prediction scoring of AT: absence of leucorrhoea and metrorrhagia, ovarian cyst larger than $5 \mathrm{~cm}$ or ovarian enlargement seen with US, vomiting and unilateral lumbar or abdominal pain lasting at least for 8 hours [2].

She also presented with one of the most recognized risk factors for ovarian torsion: ovarian hyperstimulation syndrome with consequent cystic increase in ovarian volume [3] (being the other main risk factors for ovarian torsion the hypermobility of the ovary, the presence of adnexal masses and pregnancy [2]). As it is described by the literature, right ovarian torsion occurs more frequently ( $3 / 2$ of cases) than the left one, and our case fits this recognized tendency. The right ovary is much more prone to twisting because of a longer ligament, while the left ovary is protected by the presence of the sigmoid colon which is fixer than the descending one [10]. 
For what concerns the management, as the vast majority of torsion cases occur in acute setting, sonographic examination was the first exam of choice for our patient, as it is prescribed by the literature. As in the case of our patient, however, transvaginal US is often non diagnostic; very commonly, it is only suggestive of ovarian torsion because of the missed visualization of the right ovary, which, on its turn, is highly indicative of an ovarian dislocation. Doppler study is recommended by the literature as an extension of transvaginal US for a more precise diagnostic confirmation: in the case of our patient it was not feasible because of the ovarian position. By the way US, when positive and diagnostic, usually shows a predominantly solid appearance, unilateral enlargement and adnexal masses (in $85.7 \%$ of cases); there can be also marked stromal edema and free pelvic fluid because of the arterial, venous and lymphatic occlusion caused by the adnexal twisting with the consequent parenchymal congestion and hemorrhage. On the other hand, Doppler studies, when positive, may show reduced or absent blood flow to the ovary indicating venous and arterial occlusion and thus adnexal torsion. The main problem with Doppler US is that it has been demonstrated to be normal in $60 \%$ of torsion cases and to have high specificity $(91.7 \%)$ but low sensitivity $(43.8 \%)$ with a moderate positive and negative predictive value ( $78 \%$ and $71 \%$ respectively) [4,5].

$\mathrm{MRI}$, on the other side, has to be reserved for selected cases only, presenting with subacute/chronic or recurrent presentation or for cases in which the diagnosis is unclear after US and Doppler studies [4]. Our patient fell into this second category and she actually underwent a preoperative MRI which turned out to be diagnostic.

Here the fourth point that actually makes our patient's case such an interesting object of discussion. According to what demonstrated by the updated literature, MRI is not routinely done preoperatively because of its high costs and low availability (not all structures can afford it) and the patients usually go straight to explorative laparoscopy immediately after an ambiguous US examination. The puzzling thing is that, among all these patients who undergo laparoscopy (LPS) for a suspect of ovarian torsion, only less than a half reveal to be correctly diagnosed (46.1\%) [4,5]. This means, in few words, that $54 \%$ of these patients undergo an invasive procedure with no benefit at all. In our case, instead, the patient had the chance to undergo an MRI just before heading to surgery and it turned to be of a big help; it actually confirmed the suspect making the surgical choice easier to be made and truly curative. Furthermore, MRI does not entail any radiation exposure and the risk linked to eventual contrast medium administration is very small. In conclusion, our case report aims at becoming a cause for reflection. Still today, the accuracy of the preoperative diagnosis has to be largely improved: US and Doppler studies alone are not enough in the decision making process about whether to operate or not. Therefore, further research on non-invasive preoperative tests is still needed to avoid useless surgeries: MRI has been demonstrated to be of great value and more specific serum markers could as well be of big help [5].

\section{Consent}

Written informed consent was obtained from the patient for the publication of this Case Report and of any accompanying images.

\section{Competing Interests}

The authors declare that they have no competing interests.

\section{References}

1. Nair S, Joy S, Nayar J (2014) Five year retrospective case series of adnexal torsion. J Clin Diagn Res 8: OC09-13.

2. Bider D, Mashiach S, Dulitzky M, Kokia E, Lipitz S, et al. (1991) Clinical, surgical and pathologic findings of adnexal torsion in pregnant and nonpregnant women. Surg Gynecol Obstet 173: 363-366.

3. Rackow BW, Patrizio P (2007) Successful pregnancy complicated by early and late adnexal torsion after in vitro fertilization. Fertil Steril 87: 697-712.

4. Chiou SY, Lev-Toaff AS, Masuda E, Feld RI, Bergin D, et al. (2007) Adnexal torsion: new clinical and imaging observations by sonography, computed tomography, and magnetic resonance imaging. J Ultrasound Med 26: 1289-1301.

5. Bar-On S, Mashiach R, Stockheim D, Soriano D, Goldenberg M, et al. (2010) Emergency laparoscopy for suspected ovarian torsion: are we too hasty to operate? Fertil Steril 93(6): 2012-2015.

6. Kart C, Aran T, Guven S, Karahan SC, Yulug E, et al. (2011) Acute increase in plasma D-dimer level in ovarian torsion: An experimental study. Hum. Reprod 26: 564-568.

7. Topçu HO, Topçu HO, İskender CT, Ceran U, Kaymak O, et al. (2015) Evaluation of the Diagnostic Accuracy of Serum D-Dimer Levels in Pregnant Women with Adnexal Torsion. Diagnostics 5: 1-9.

8. Humaidan P, Papanikolaou EG, Kyrou D, Alsbjerg B, Polyzos NP, et al. (2012) The luteal phase after GnRH-agonist triggering of ovulation: present and future perspectives. Reprod Biomed Online 24: 134-141.

9. Devroey P, Polyzos NP, Blockeel C (2011) An OHSS-Free Clinic by segmentation of IVF treatment. Hum Reprod 26(10): 2593-2597.

10. Tsai HC, Kuo TN, Chung MT, Lin MYS, Kang CY, et al. (2015) Acute abdomen in early pregnancy due to ovarian torsion following successful in vitro fertilization treatment. Taiwan J Obstet Gynecol 54: 438-441. 